\title{
Research on the method of feature extraction based on ARM platform
}

\author{
Dezhi Zheng ${ }^{1, \text { a }}$, Kunming Wang ${ }^{2, b^{*}}$, Jialin Cheng ${ }^{3, c}$, Shangchun Fan ${ }^{4, d}$ \\ ${ }_{1,2,3,4}$ School of Instrument Science and Opto-electronics Engineering, Beijing University of \\ Aeronautics and Astronautics, Beijing 100191, China \\ azhengdezhi@buaa.edu.cn, ${ }^{b^{*}}$ hawgvit@aliyun.com, ${ }^{c}$ chengjialin@163.com, ${ }^{d}$ fsc@buaa.edu.cn
}

Keywords: Colloidal gold reagent; Quantitative detection; Feature extraction; Least square method; Weight function;

Abstract: Detection method of colloidal gold immunochromatography assay concentration using colorimetric principle, when tested in liquid reagent on antigen and antibody response, reagent detection area change color, and compared to standard color grade then the concentration can be qualitative analysis. At present, the concentration of colloidal gold test strip is mostly semi quantitative, so the application scope is limited. This paper designs an image shooting based on embedded ARM platform application which processor using ARM v7 architecture, equipped with WINCE system, the OV9650 CMOS (Complementary Metal Oxide Semi-conductor) image sensor to obtain quantitative colloidal gold test strip image information, while setting manual white balance, exposure time, manual gain, then extracting ruler window setting image cut. And we can judge and recognize the characteristics of the detection regions, through using the least square method to further reduce the error, and for the effective feature region of the most value and area value, design weight function, to improve the repeatability of measurement results. The paper makes a quantitative analysis of the image of the reagent, and the repeatability error can reach $\pm 1 \%$. Through the analysis of the research the results are in accord with the design requirements. The design and algorithm of this paper have some practical value.

\section{Introduction}

The tradition way of detecting colloidal gold immunochromatography concentration applies colorimetric principle. When antigen in the testing liquid and antibody on reagent react, the detection region on reagent changes color; visual qualitative analysis is performed by comparing with standard color $_{\text {card }}{ }^{[1]}$. Extensively used in areas such as biochemical preparation, food and drugs, and medical examination and inspection ${ }^{[2]}$, most colloidal gold reagent uses semi-quantitative measurement ${ }^{[3]}$, putting limitation in its application scope. Therefore, image shooting is used to accurately detect the colloidal gold reagent concentration instead of traditional visual analysis.

Thus, this paper designs image shooting based on embedded ARM platform, miniaturizes the device, making it portable and easy to exploit. At the same time, calculation method of image feature extraction is enhanced, improving testing reagent accuracy, stability, and sensitivity, expanding testing area, enhancing testing efficiency, providing prospects for development of colloidal gold reagent quantitative detection, and having wide application value.

\section{Image shooting and image feature}

This paper employs ARM v7 architecture processor equipped with WINCE system, and exploits application program and writes arithmetic through VS2008. Camera used for image shooting is 1.3 megapixel color camera, model OV9650 from OmniVision company. This camera is small and has low operating voltage; users have complete control over image quality, data format, and transmission mode. All image processing functions including Gamma Curve, white balance, saturation level, and Chroma can be programmed through SCCB (Serial Camera Control Bus) interface. The image sensor applies unique sensor technology, from reducing or eliminating optics or electronic defects such as fixed pattern noise, smearing, and blooming, image quality can be improved and clear and stable color image can be obtained. 
Since image shooting takes place in darkroom, supplying relatively stable illuminate, this paper sets parameters such as manual white balance, manual gain, and manual exposure time in register according to experimental environment, stabilizes results shooting under repetitive shooting, having better repeatability and stability comparing to automatic white balance, automatic gain, and automatic exposure. Besides, windowing function is used, making output image containing only feature area needed, decreasing stored data, reducing original image from $2.5 \mathrm{M}$ bytes to $128 \mathrm{~K}$ bytes, lowering the image shooting time, improving ARM processing speed, and saving final BMP image output in RGB565 format for the following feature extraction using image arithmetic.

Image feature extraction and identification have significant theorical and practical meaning ${ }^{[4]}$. Fig. 1 demonstrates four images to be feature extracted, in which the region selected by thick black box is quality control area( $\mathrm{C}$ area $)$, characterizes the effectiveness of test; the region in thin box is characterization test area ( $\mathrm{T}$ area), the feature region of the proposed algorithm, distinct concentration value is distinguished from determine the color change in this region. In Fig. 1, feature value from large to small is shown accordingly from left to right.

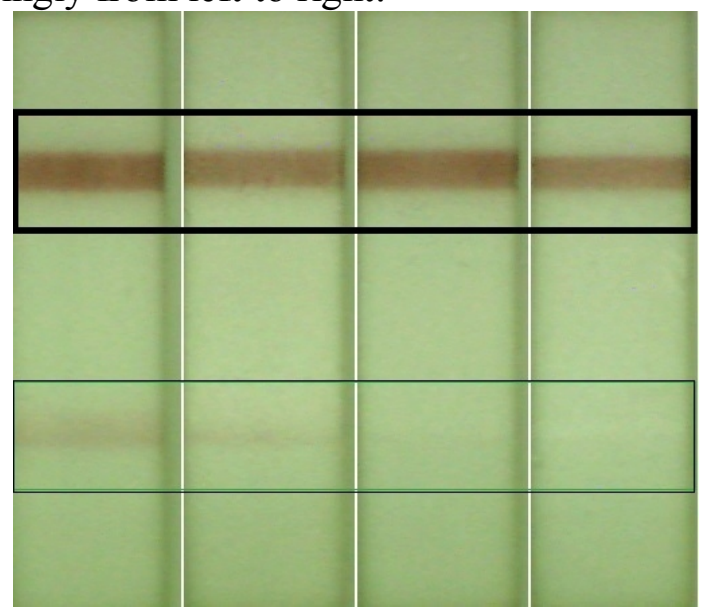

Fig. 1 Four different concentration reagent images

\section{Image feature extraction algorithm}

In general, a favorable image feature extraction method should have the following two qualitie $^{[5]}$ : first, for the determined image feature, appropriate information should be supplied, accurately approximating geometric matching conversion. Second, they should be identified by computer algorithm easily, making the whole feature extraction process automatic. To extract image feature better, the proposed algorithm first pre-processing the image, including color image gray image conversion and mean filter.

Color image conversion to gray image: converting color image to gray image, not only reduces image storage space, but also significantly improves ARM processing time! Gray image eliminates image personality, reflecting more of image display ${ }^{[6]}$ !

Most color image apply RGB mode, every color in the image is consists of red, green, and blue base color. Therefore, when storing color information, because it is saved in RGB565 format, conversion from RGB565 to RGB888 occurs first, meaning three separate bytes are needed to store $\mathrm{R}, \mathrm{G}$, and $\mathrm{B}$. Yet gray image requires only grey level to describe image, so one byte is sufficient. Graying process of color image is to transform R, G, and B to one gray level value using different methods. The human eye has distinct sensitivity for different colors, according to scientific experiment, it is most sensitive to green, followed by red and then blue. Therefore, the following function is used to obtain reasonable gray image by weighing average of RGB three components:

$$
f(x, y)=0.30 R(x, y)+0.59 G(x, y)+0.11 B(x, y)
$$

Mean filter: mean filter is the most basic smooth filter, also called linear filter, the primary method applied is neighborhood averaging, with replacing each pixel value of original image with average value as its fundamental. Let gray level of center pixel $(i, j)$ be $f(i, j)$, set A represents all 
pixel points in window, $\mathrm{L}$ is the number of pixel in set $\mathrm{A}$. by applying mean filter (neighborhood averaging), the corresponding gray value of center pixel $f(i, j)$ is:

$$
g(i, j)=\frac{1}{\mathbf{L}} \sum_{(x, y=\mathrm{L})} \mathrm{f}(\mathrm{x}, \mathrm{y})
$$

Replacing original gray value with average value of window pixel $f(i, j)$. Mean filter can restrain noise effectively at a certain extent, and its algorithm time is short ${ }^{[7]}$.

Pre-processing original image, grey level image, and gray level after filtering are shown in Fig. 2, feature curve is shown in Fig. 3. Gray image feature curve is smoother after filtering, reducing errors effectively.

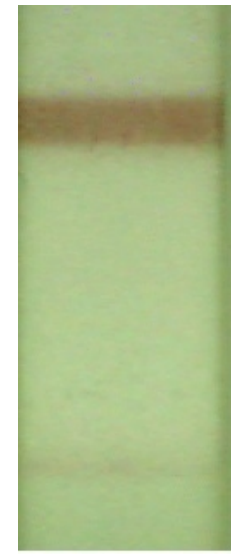

a) Original image

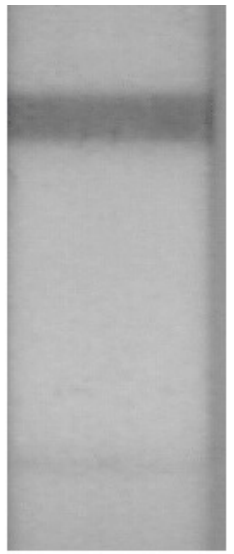

b) Gray image

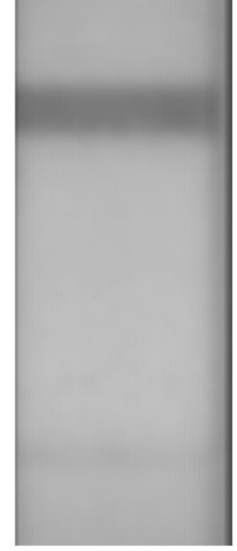

c) Gray image after mean filtering

Fig. 2, original pre-processing image, gray level image, and gray image after filtering

Flow Chart of image feature extraction algorithm are shown in Fig. 4.

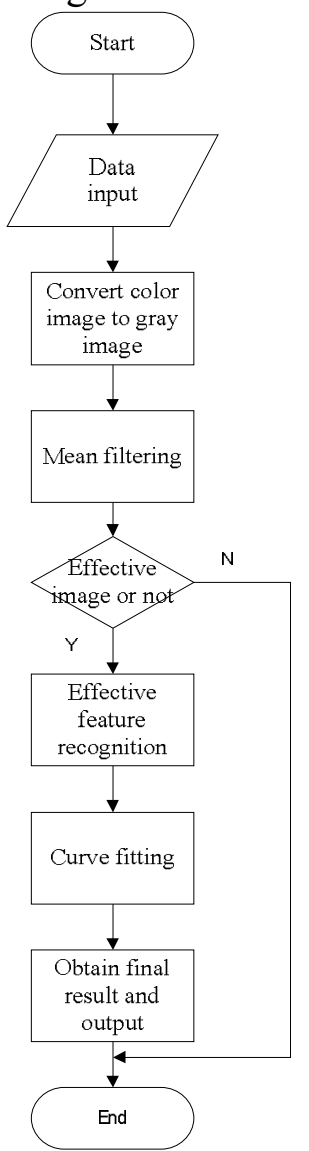

Fig. 4 Flow Chart of algorithm 
1) Judging the effectiveness of image: by extracting feature value in $C$ region, and judging if the number falls within the effective range, the detection is invalid if not within the range, subsequent algorithm should be abandoned and the invalidation should be reminded. If the number falls within the effective range, then proceed to next step.

2) Effective feature region recognition: since the space between quality control area $\mathrm{C}$ and test area $\mathrm{T}$ is consistent of reagent within the same batch, control area $\mathrm{C}$ can not only be used to determine the effectiveness of test, but also to confirm the range of $\mathrm{T}$ area. This algorithm first sets threshold value based on actual measured value to confirm the initial position of $\mathrm{C}$ area, then, from the initial position of $\mathrm{C}$ area to determine the range of $\mathrm{T}$ area, which is the effective feature area. Position errors due to reagent machinery installation can be effectively reduced applying this method.

3) Curve fitting: least square method is the most commonly used method for curve fitting. It is not only the most important statistical method in the $19^{\text {th }}$ century, but also known as the soul of mathematical statistics. Several branches of mathematical statistics such as relevant regression analysis, variance analysis, and linear model theory are all theoretically based on least square method. For further development and to correct deficiency of such method, many modern mathematical branches are derived from least square method ${ }^{[8]}$. Just like what S.M. Stigler said, 'least square method to mathematical statistics is like calculus to mathematics" ${ }^{, 9]}$.

Curve fitting by applying least square method:

For the $\mathrm{n}$ data points already known $\left(\mathrm{x}_{\mathrm{i}}, \mathrm{y}_{\mathrm{i}}\right)(\mathrm{i}=1,2, \ldots, \mathrm{n}-1)$, calculation of least square fitting polynomial to the power of $(\mathrm{m}-1)$ is needed

$$
p_{m-1}(x)=a_{0}+a_{1} x+a_{2} x^{2}+\cdots+a_{m-1} x^{m-1}
$$

In it, $\mathrm{m} \leq \mathrm{n}, \mathrm{n} \leq 20$. Calculate coefficients $a_{0}, a_{1}, \ldots, a_{m-1}$ in fitting polynomial.

Fitting polynomial is the linear combination of orthogonal polynomial $Q_{j}(x)(\mathrm{j}=0,1, \ldots, \mathrm{m}-1)$ :

$$
P_{m-1}(x)=c_{0} Q_{0}(x)+c_{1} Q_{1}(x)+c_{2} Q_{2}(x)+\cdots+c_{m-1} Q_{m-1}(x)
$$

$Q_{j}(x)$ can be constructed by the following recursion formula:

$$
\begin{aligned}
& Q_{0}(x)=1 \\
& Q_{1}(x)=\left(x-\alpha_{1}\right) \\
& \left.Q_{j+1}(x)=\left(x-\alpha_{j+1}\right) Q_{j}(x)-Q_{j-1}(x), 0=1,2, \ldots, \mathrm{m}-1\right)
\end{aligned}
$$

Let

$$
d_{j}=\sum_{i=0}^{n-1} Q_{j}^{2}\left(x_{i}\right)_{j}=0,1, \ldots, \mathrm{m}-1
$$

Then

$$
\begin{aligned}
& \alpha_{j+1}=\frac{1}{d_{j}} \sum_{i=0}^{n-1} x_{i} Q_{j}{ }^{2}\left(x_{i}\right), j=0,1, \ldots, \mathrm{m}-2 \\
& \beta_{j}=d_{j} / d_{j-1}, j=0,1_{1} \ldots, \mathrm{m}-2
\end{aligned}
$$

Can prove that, polynomial function $Q_{j}(x)(\mathrm{j}=0,1, \ldots, \mathrm{m}-1)$ from recursion formula above is quadrature. According to least square theory, obtain:

$$
c_{j}=\frac{1}{d_{j}} \sum_{i=0}^{n-1} y_{i} Q_{j}\left(x_{i}\right), j=0,1, \ldots, \mathrm{m}-1
$$

Can be converted to normal polynomial to the power of m-1

$$
P_{m-1}(x)=a_{0}+a_{1} x+a_{2} x^{2}+\cdots+a_{m-1} x^{m-1}
$$

Polynomial coefficient $a_{0}, a_{1}, \ldots, a_{m-1}$ can be calculated using iteration.

Focusing on the actual image, this algorithm first chooses 80 pixels contained in feature area to curve fitting, then reduces the feature range to 42 pixels in order to minimize error.

4) Obtaining result: besides corresponding feature region area $(\mathrm{S})$, this algorithm also introduces feature region most value $(\mathrm{M})$, final result $(\mathrm{V})$ is the value from weighting $(\mathrm{W})$ factor between feature region most value and feature region area. The weight here is set according to actual most value measured and feature region area. Weight function used for this algorithm is: 


$$
\mathrm{V}=\mathrm{M}^{*}(\mathrm{~W}-\mathrm{M}) / \mathrm{W}+\mathrm{S} *[1-(\mathrm{W}-\mathrm{M}) / \mathrm{W}]
$$

\section{Test and result}

Applying the proposed algorithm to extract feature for the four distinct concentration images in Fig. 1, gray level curve graph within 80 pixel range obtained by feature region determination algorithm is shown in Fig. 5 (from left to right in Fig. 1 correspond to color red, green, blue, and yellow), as shown to the left of Fig. 5, this algorithm can effectively distinguish feature region of different concentration, the 80 pixels in the image contain effective feature of different concentration accurately (the concave down portion in the diagram covers 42 pixels). From curve fitting using 8 level least square method, gray level curve diagram of 80 pixels is obtained (from left to right in Fig. 1 correspond to color red, green, blue, and yellow), as shown to the right of Fig. 5, image after fitting effectively reduces data fluctuate error, smoothing the curve, and the result is exceptional for extracting feature from low concentration reagent.
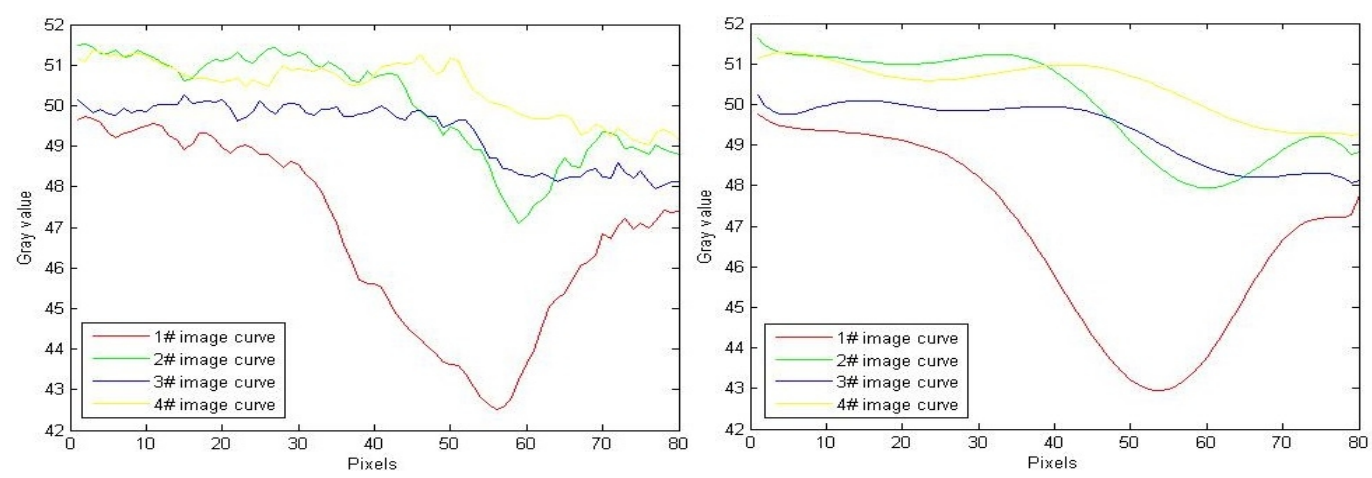

Fig. 5, pre-fitting and post-fitting curve diagram for four different reagent concentrations

Feature extraction is performed for eight different concentration images applying proposed algorithm, feature results see Fig. 6. From Fig. 6, the proposed algorithm has better resolving power for both higher and lower concentration, and the result agrees with high and low concentration trend.

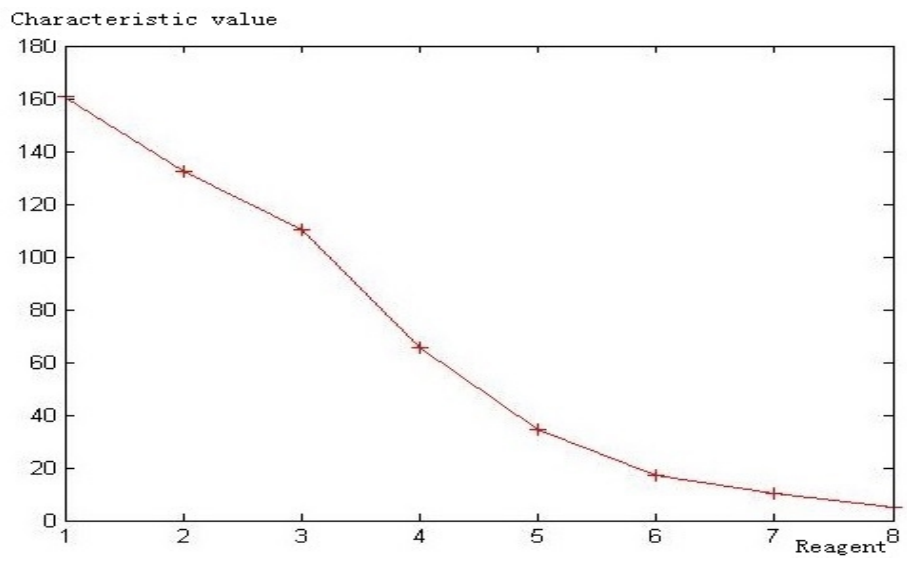

Fig. 6, feature result of eight concentration image

From the gray level of 80 pixel after fitting shown in Fig. 5 to confirm the actual corresponding 42 pixels valid feature region, experiment shoots the same image repeatedly, obtains result as shown in Table 1: 
Table 1, feature result of eight concentration image

\begin{tabular}{|c|c|c|c|c|c|}
\hline No. & First & Second & Third & Forth & Standard deviation \\
\hline $1 \#$ & 160.8 & 160.8 & 161.2 & 161.4 & 0.32 \\
\hline $2 \#$ & 136.8 & 136.6 & 137.1 & 137.3 & 0.32 \\
\hline $3 \#$ & 110.2 & 110.9 & 111.1 & 110.6 & 0.39 \\
\hline $4 \#$ & 61.7 & 60.0 & 61.3 & 61.1 & 0.75 \\
\hline $5 \#$ & 33.6 & 34.6 & 35.7 & 35.3 & 0.94 \\
\hline $6 \#$ & 20.1 & 20.7 & 20.4 & 21.0 & 0.39 \\
\hline $7 \#$ & 9.7 & 10.0 & 10.3 & 10.3 & 0.27 \\
\hline $8 \#$ & 5.4 & 4.8 & 5.6 & 4.8 & 0.43 \\
\hline
\end{tabular}

As shown in Table 1, results among different shoots has errors, the possible cause is light fluctuation and exposure time error of OV9650 image shooting sensor. Yet abundant experiments have proved that dispersion is small for the proposed algorithm, all errors are within \pm 1 range

\section{Conclusion}

The design of this paper actualizes feature detection of quantitative colloidal gold reagent based on ARM image processing system, using OV9650 model CMOS image sensor to collect original image, from setting manual white balance, manual exposure time, and manual gain to extract ruler window setting image cut. Through recognition and determination of feature region, errors are further reduced by least square method curve fitting, and weighting (W) factor between feature region most value and feature region area is introduced in order to get final results. Quantitative analysis is performed for reagent image, repeatability error achieved $\pm 1 \%$. Comprehensive experimental results show that, the system accomplishes feature detection of quantitative colloidal gold reagent, and its repeatability is high.

\section{ACKNOWLEDGEMENTS}

This work was supported in part by the foundation item of Chinese National High-tech Researc $\mathrm{h}$ and Development Program (2014BAF08B01). It is also supported by TransCentury Training Prog ramme Foundation for the Talents of Humanities and Social Science by the State Education Commi ssion and the Yong Talent Program of the Central Universities Co-building Project of Beijing.

\section{References}

[1] Abe C, Hirano K, Tom iyama T. Simple and rapid identification of mycobacterium tuberculosis complex by immunochromatography assay using anti-MPB64 monoclonal antibodies[J]. Journal of Clinical Microbiology,1999,37(11):3693-3697

[2] James Camey, Helen Braven, Joanna Seal, et al. Preset and future application of gold in rapid assay[J]. IVDTechnology,2006(5):41-44

[3] Wang Shuai, Zheng Dezhi, Fan Shangchun. Detecting method of quantitative collodialgold test strip concentration [J]. Journal of Beijing university of aeronautics and astronautics , 2009 , $8: 942-945$

[4] Wen-Sheng Tang, Shao-Hua Jiang, Shu-Lin Wang, Gray scale potential: A new feature for sparse image[J]. Neurocomputing, 2013, Vol.116, pp.112-121

[5] Peihua Qiu, Chen Xing, Feature based image registration using non-degenerate pixels[J]. Signal Processing, 2013, Vol.93 (4)

[6] S. Rakshit, A. Ghosh, B. Uma Shankar, Fast mean filtering technique (FMFT)[J]. Pattern 
Recognition, 2006, Vol.40 (3), pp.890-897

[7] Jiang Weiwei, Lu Changhua etc.. Improved adaptive switching median filter [J]. Journal of instrument and meter.2011,32(6):137-139

[8] Jia Xiaoyong, Xu Chuansheng, The invention and way of thinking on least squares[J]. Journal of Northwest University(Natral Science Editon), 2006, 6: 507-511

[9] LANCASTER H O. Encyclopedia of statistical science [J]. New York :W iley,1988. 